\title{
Noise Shaping by Interval Correlations Increases Information Transfer
}

\author{
Maurice J. Chacron, Benjamin Lindner, and André Longtin \\ Department of Physics, University of Ottawa, 150 Louis Pasteur, Ottawa, Canada K1N-6N5
}

(Received 20 June 2003; published 25 February 2004)

\begin{abstract}
The influence of intrinsic firing interspike interval correlations on the noise spectrum and information transfer is studied. This is done through the comparison of two simple firing models, one of which is a renewal process while the other displays interval correlations. These correlations are shown to shape the spike train power spectrum and, in particular, to decrease the noise power at low frequencies. Linear response theory and numerical simulations reveal how this shaping can increase the transmission of information about a time-varying signal. Our results are relevant to the analysis of nonrenewal point processes and signal detection in physics and biology.
\end{abstract}

DOI: 10.1103/PhysRevLett.92.080601

There has been much interest over the past decades in studying escape problems from metastable states [1]. In several cases, the output is a sequence of spike responses which can be described by a point process. Examples include lasers [2], photodetection [3], and neural spike trains $[3,4]$. In many systems, one is concerned with the statistics of the interspike intervals (ISIs) [1,5]. Most studies of excitable systems deal with first order ISI statistics such as their probability distribution. Point processes in which all second and higher order ISI correlations are zero are called renewal processes [6]. However, there is experimental evidence that many excitable systems are nonrenewal. This is the case for many neurons responding to sensory stimuli [7] including electroreceptive neurons [8,9] in electric fish that must respond to low frequency stimuli caused by prey [10]. Their firing is nonrenewal since, even in the absence of an input signal, they exhibit intrinsic nonzero ISI serial correlation coefficients defined as

$$
\rho_{j}=\frac{\left\langle\left(I_{i+j}-\left\langle I_{i}\right\rangle\right)\left(I_{i}-\left\langle I_{i}\right\rangle\right)\right\rangle}{\left\langle\left(I_{i}-\left\langle I_{i}\right\rangle\right)^{2}\right\rangle},
$$

where $\left\{I_{i}\right\}$ denotes the ISI sequence, $j$ is the lag, and the average $\langle\ldots\rangle$ is performed over index $i$.

It has been shown that a leaky integrate-and-fire neuron with a dynamical threshold (LIFDT) could produce a spike train that displayed negative ISI correlations $[9,11,12]$. The action potential threshold is highly variable in neurons and can increase under repetitive firing [13]. Furthermore, numerical simulations suggest that negative ISI correlations improve the detectability of weak signals [12]. However, the actual mechanism by which intrinsic negative ISI correlations may increase information transfer is presently unknown due to the LIFDT model's complexity and to the memory carried by the threshold variable. The effects of such correlations on the spike train power spectrum and on signal transmission are the focus of our Letter. In particular, we demonstrate that this information transfer increase occurs through a reduction in the low frequency noise. Such
PACS numbers: 05.40.-a, 87.17.Nn, 87.19.La, 87.19.Bb

reductions are of great interest in a variety of applications involving nonlinear oscillators, such as in Josephson junctions [5,14]. Noise shaping effects were also observed in neural networks with feedback [15], even though the dynamics were renewal.

In order to analytically explore the role of negative correlations, we use two simple spiking models, A and B. We show that models A and B have identical first order ISI statistics as well as identical linear response with respect to external stimulation. Both models are perfect integrators of the input, i.e., the observable output (e.g., the voltage across the nerve membrane for a neuron) is governed by

$$
\dot{v}(t)=\mu+s(t),
$$

where $\mu$ is a positive constant bias and $s(t)$ is a timevarying signal. Also, in both models, the threshold is drawn randomly from a uniform distribution on $\left[\Theta_{0}-D\right.$, $\left.\Theta_{0}+D\right]$; whenever the voltage reaches this (piecewise constant) threshold, a spike is generated and a new threshold is drawn (this is similar to previously proposed models [16]).

The models differ in their reset rules for the voltage. For model A, the voltage is decremented after spiking by the constant $\Theta_{0}$ (which is the mean threshold value). Clearly, this kind of reset carries memory of the previous threshold value, thus correlating subsequent ISIs; consequently, model A generates a nonrenewal spike train. This can be seen for $s(t) \equiv 0$ considering the subintervals $U_{j}$ and $V_{j}$ with $I_{j}=U_{j}+V_{j}$ in Fig. 1: for obvious reasons we have $V_{j}+U_{j+1}=\Theta_{0} / \mu$; hence subsequent intervals are correlated via these subintervals. It is straigthforward to show that the ISI correlation coefficient is $\rho_{l}=\delta_{l, 0}-$ $\delta_{l, 1} / 2$, which is a simplified description of the negative correlation displayed by either real neurons and the more complicated LIFDT model $[9,12]$. Further, note that the distribution of reset values equals that of the threshold values except for the mean; i.e., reset values are distributed uniformly in $[-D, D]$.

For model B we choose a random reset $\theta_{r}(t)$ drawn from the uniform distribution $[-D, D]$. which is the same 
(a)

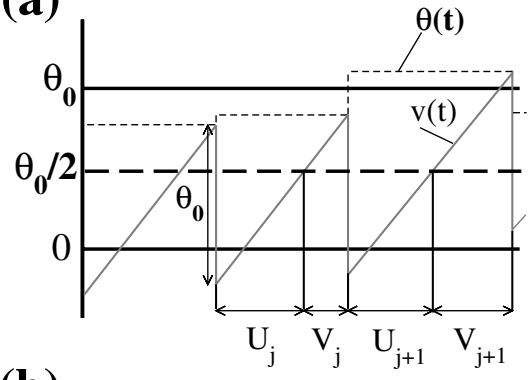

(b)

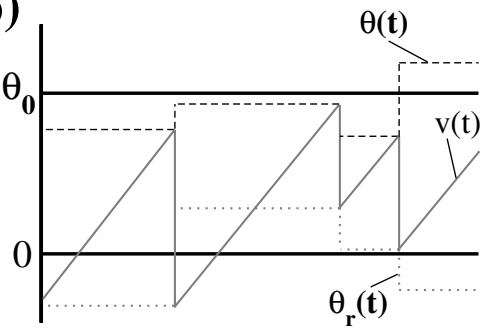

FIG. 1. (a) The dynamics of model A. Shown are the voltage $v(t)$ (solid line) which rises linearly with slope $\mu$ and threshold $\theta(t)$ (dashed line) under spontaneous activity $[s(t)=0]$. When $v(t)=\theta(t)$, the voltage is decremented by $\theta_{0}$ while the threshold is reset to a uniform random value in the interval $\left[\theta_{0}-D, \theta_{0}+D\right]$. Also shown are the ISI $I_{j}$ that can be divided into the subintervals $U_{j}$ and $V_{j}$ such that $I_{j}=U_{j}+V_{j}$. (b) The dynamics of model $\mathrm{B}$ are identical to those of model $\mathrm{A}$ except that the voltage is reset to a uniform random value $\theta_{r}(t)$ (dotted line) in the interval $[-D, D]$. This eliminates memory and thus ISI correlations.

as for model A. This leads to completely independent ISIs, i.e., to a renewal point process [6] with $\rho_{l}=\delta_{l, 0}$.

We first focus on the spontaneous activity [i.e., $s(t)=$ $0]$ of both models since most neurons that display negative ISI correlations are known to be spontaneously active in experiments [7,8]. Since the distributions of initial (reset) and final (threshold) values are the same for A and B, we obtain in both cases the same ISI density, a "triangular" function [cf. inset of Fig. 2(a)] that results from convolution of two independent uniformly distributed random numbers. Furthermore, one can determine the Fourier transforms of the density of the $n$th order interval (i.e., the sum of $n$ subsequent intervals). By using these together with well-known formulas from the theory of point processes [17], one can determine the power spectra of spike trains for both models [18]. We obtain

$$
S_{A 0}(f)=\frac{1}{\langle I\rangle}-\frac{\sin ^{2}(\beta f)}{(\beta f\langle I\rangle)^{2}}\left[\langle I\rangle-\sum_{n=-\infty}^{\infty} \delta\left(f-\frac{n}{T}\right)\right],
$$

$S_{B 0}(f)=\frac{\left[(\beta f)^{4}-\sin ^{4}(\beta f)\right] /\langle I\rangle}{(\beta f)^{4}-2(\beta f)^{2} \sin ^{2}(\beta f) \cos (2 \pi\langle I\rangle f)+\sin ^{4}(\beta f)}$,

where $\beta=2 \pi D / \mu$ and the mean ISI is given by $\langle I\rangle=$ $\Theta_{0} / \mu$. Both spectra are shown in Fig. 2(b) and con-
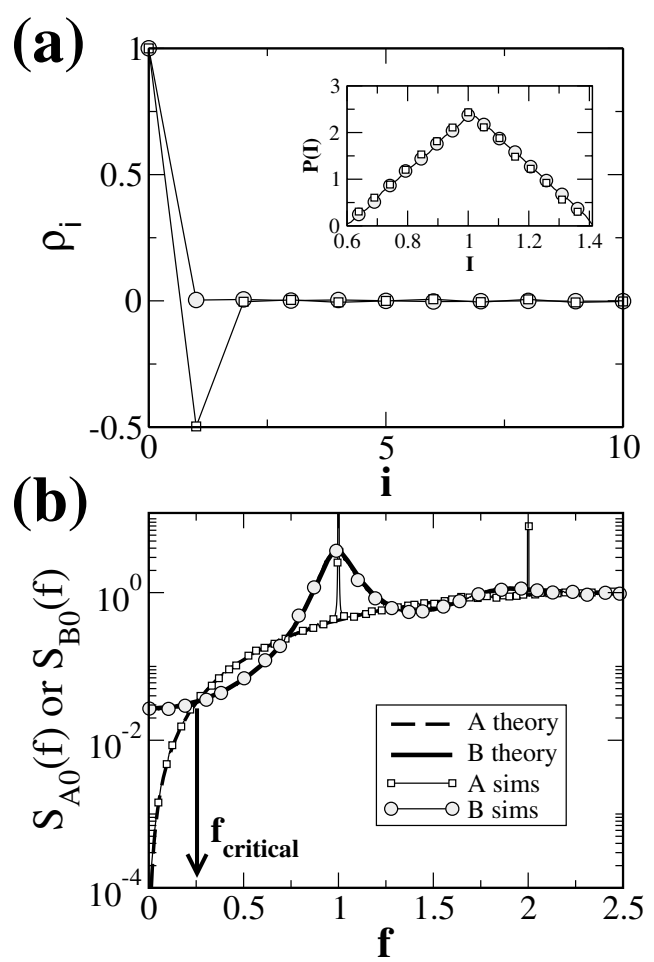

FIG. 2. Spontaneous activity of both models. (a) The ISI correlations coefficients $\rho_{j}$ obtained numerically with model A (open squares) and model B (grey circles) are in excellent agreement with theoretical predictions (see text). (b) The power spectra obtained numerically with model A (open squares) and model B (grey circles) are in respective good agreement with the theoretical expressions (dashed and solid lines ) given by Eqs. (3) and (4). The two spectra first intersect at $f_{\text {critical }}$ (arrow). Simulated spectra were computed from $10^{5}$ action potentials and averaged over nonoverlapping segments. Parameter values for numerical simulations were $\theta_{0}=1, \mu=1, D=0.2$, and $s(t) \equiv 0$.

firmed by results of simulations. The spectrum of model A contains $\delta$ peaks at the inverse mean ISI and higher harmonics of this frequency. More interesting is the fact that the continuous background in Eq. (3) goes to zero for vanishing frequency. Both the $\delta$ peaks and the vanishing spectrum at $f \rightarrow 0$ can be understood by means of the negative correlations of model A. The power spectrum at $f=0$ is related to the ISI correlation coefficient by $C V^{2}\left(1+2 \sum_{i=1}^{\infty} \rho_{i}\right) /\langle I\rangle$ [6] where the coefficient of variation of the interspike interval is finite and the same for both models. Using this relation leads to a vanishing power spectrum at $f=0$ for model A. Moreover, the ISI correlations shape the background spectrum rather than reduce the total noise power.

We investigate the consequences of these differing power spectra on information transfer by both models by studying their encoding capabilities using zero-mean band-pass limited white noise stimuli $s(t)$. Such stimuli have a power spectrum $S_{\text {st }}(f)=\alpha$ if $|f|<f_{c}$ and $S_{\mathrm{st}}(f)=0$ otherwise. Here $f_{c}$ is the cutoff frequency. 
Such stochastic stimuli are widely used to assess signal transmission in neurons [4,19]. Information theory $[19,20]$ is used to quantify the amount of information that a system can transmit about these signals. For systems driven with Gaussian white noise stimuli, a lower bound on the mutual information rate can be calculated [4] as

$$
\mathcal{M}_{\mathrm{A}, \mathrm{B}}=-\int_{0}^{f_{c}} d f \log _{2}\left[1-C_{\mathrm{A}, \mathrm{B}}(f)\right],
$$

where $C_{\mathrm{A}, \mathrm{B}}(f) \equiv\left|X_{\mathrm{A}, \mathrm{B}}(f)\right|^{2} /\left[S_{\mathrm{A}, \mathrm{B}}(f) S_{\mathrm{st}}(f)\right]$ is the coherence function; $X_{\mathrm{A}, \mathrm{B}}(f), S_{\mathrm{A}, \mathrm{B}}(f)$ are, respectively, the cross spectrum between spike train and signal and the power spectrum of the spike train in the presence of the signal.

An evolution equation for the probability density of the voltage variable has been derived and solved in the presence of a periodic signal. This solution enables us to compute the susceptibility and to derive the power and cross spectra of the spike train in the presence of a weak signal using linear response theory [21]. We obtain

$$
\begin{aligned}
& S_{\mathrm{A}, \mathrm{B}}(f)=S_{\mathrm{A} 0, \mathrm{~B} 0}(f)+\left(\frac{\theta_{0}}{\mu}\right) S_{\mathrm{st}}(f) ; \\
& X_{\mathrm{A}, \mathrm{B}}(f)=\frac{\theta_{0}}{\mu} S_{\mathrm{st}}(f) .
\end{aligned}
$$

Note that the fact that the susceptibility does not depend on frequency is a consequence of the specific noise implementation in our model [21].

We thus obtain

$$
C_{\mathrm{A}, \mathrm{B}}(f)=\left[1+\frac{\mu^{2} S_{\mathrm{A}, \mathrm{B}}(f)}{\theta_{0}^{2} S_{\mathrm{st}}(f)}\right]^{-1} .
$$

The only difference in $C_{\mathrm{A}, \mathrm{B}}(f)$ for models $\mathrm{A}$ and $\mathrm{B}$ is the baseline power spectrum $S_{\mathrm{A} 0, \mathrm{~B} 0}(f)$. The coherence is inversely proportional to $S_{\mathrm{A} 0, \mathrm{~B} 0}(f)$ which can thus be considered as a background noise spectrum that will limit the information transmission capacity. Our results demonstrate that negative ISI correlations shape the baseline spectrum and lower its value at low frequencies up to a critical frequency $f_{\text {critical }}$ [Fig. 2(b)]. This results in $C_{\mathrm{A}}(f)>C_{\mathrm{B}}(f)$ for low $f$. This is verified in Figs. 3(a) and 3(b) by comparing the predictions from Eq. (7) to numerical simulations. Theoretical predictions are in excellent agreement with numerical simulations for both models. Moreover, $C_{\mathrm{A}}(f)$ approaches 1 as $f \rightarrow 0$ while $C_{\mathrm{B}}(f)$ is always less than 0.1. The former is a direct consequence of $S_{\mathrm{A} 0}(f)$ approaching 0 for $f \rightarrow 0$.

Figure 4(a) compares the mutual information rate $\mathcal{M}$ computed from Eq. (5) to the one obtained from numerical simulations as a function of $f_{c}$. As expected, $\mathcal{M}$ is higher for model A than for model B. The difference $\Delta \mathcal{M} \equiv \mathcal{M}_{\mathrm{A}}-\mathcal{M}_{\mathrm{B}}$ shows a global maximum as a function of $f_{c}$. This is similar to previously reported numeri-
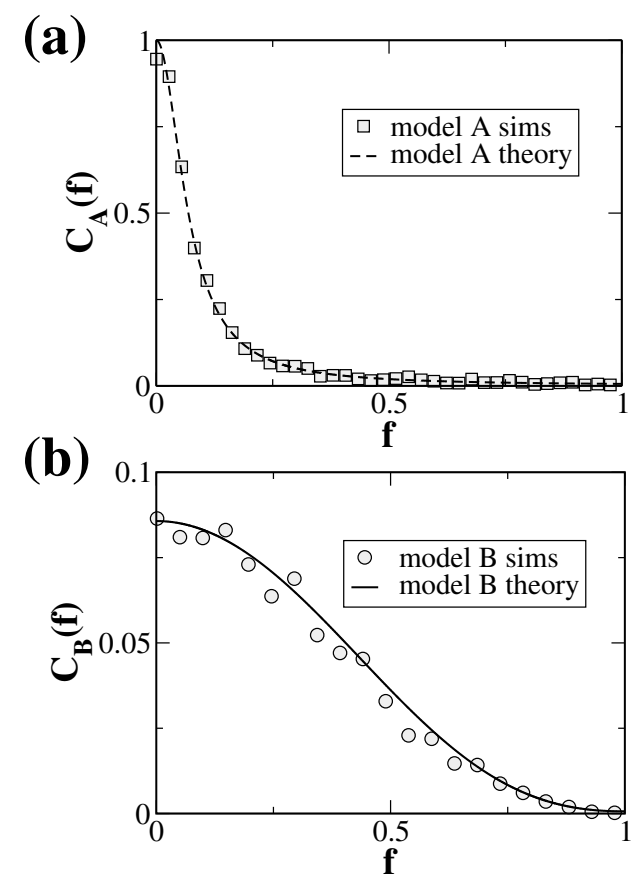

FIG. 3. (a) Coherence curves obtained numerically from model A (grey squares) and theoretically (dashed line) from Eq. (7). (b) Coherence curves obtained numerically from model B (grey circles) and theoretically (solid line) from Eq. (7). Other parameters were $\alpha=0.0025$ and $f_{c}=2$.

cal results [12] displays a frequency preference for the increase in mutual information rate. This maximum occurs for $f_{c}=f_{\text {critical }}$ [21].

Figure 4(b) reveals the effects of varying the signal intensity $\alpha$. As expected, the agreement between the linear response theory and numerical simulation is excellent for both models at low $\alpha$. However, differences occur for higher $\alpha$ as nonlinear effects become important. Numerical simulations reveal that $\Delta \mathcal{M}$ decreases as $\alpha$ increases. This is to be expected as $S_{\mathrm{st}}(f) \gg S_{\mathrm{A} 0, \mathrm{~B} 0}(f)$ and the presence or absence of background noise is of little consequence. Thus, the increase in mutual information rate seems to be greatest for lower intensity stimuli. Linear response theory will fail for large $f_{c}$ as it is sensitive to the signal variance $\left\langle\Delta s^{2}\right\rangle=2 \alpha f_{c}$ [21].

We have furthermore verified the results obtained for models A and B by actual variation of the action potential threshold in a nonrenewal manner vs a purely stochastic variation in a biologically realistic Hodgkin-Huxley model. Our results show that the modification in a nonrenewal manner can give rise to negative ISI correlations as well as greater information transfer and will be presented elsewhere [21].

In summary, we have compared two simple models, one of which displayed negative ISI serial correlations and one of which did not. Both models had identical first order ISI statistics and linear response, ensuring a fair comparison without further differences (e.g., a difference 

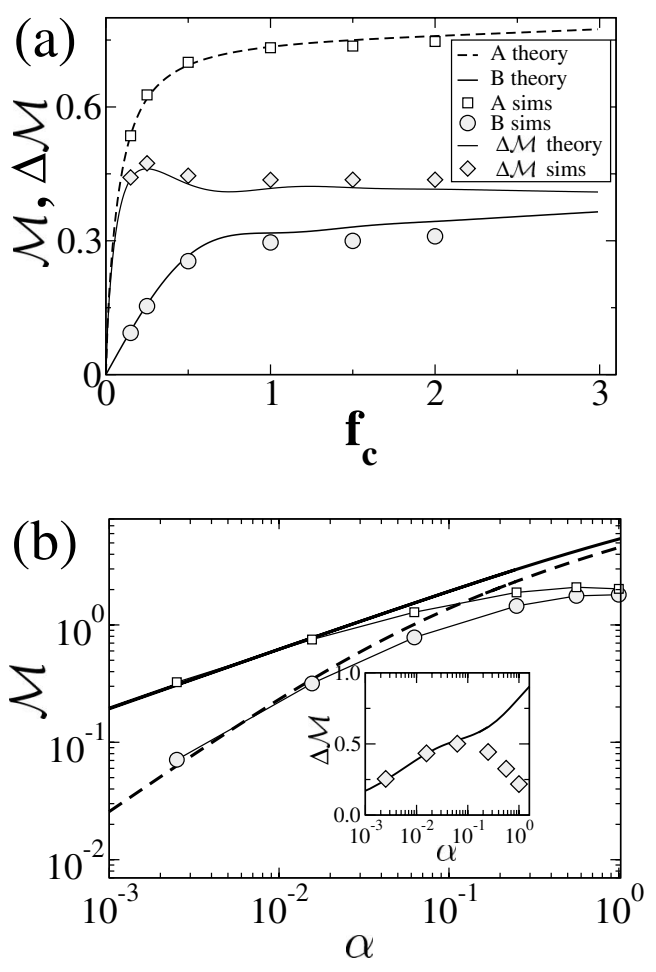

FIG. 4. (a) Mutual information rates versus cutoff frequency $f_{c}$. The numerical simulations are in respective good agreement (open squares and grey circles) with theoretical predictions (dashed and solid lines). The difference in mutual information $\Delta \mathcal{M} \equiv \mathcal{M}_{\mathrm{A}}-\mathcal{M}_{\mathrm{B}}$ displays a maximum as a function of $f_{c}$. We used $\alpha=0.0156$. (b) Mutual information rates as a function of $\alpha$. Numerical simulations and linear response theory show an increase in both $\mathcal{M}_{\mathrm{A}}$ and $\mathcal{M}_{\mathrm{B}}$ as a function of $\alpha$. Linear response fails for high $\alpha$. The inset shows a plot of $\Delta \mathcal{M}$ as a function of $\alpha$. Numerical simulations (grey diamonds) predict a maximum in $\Delta \mathcal{M}$ as a function of $\alpha$ while the linear response theory (black line) predicts an increase. We used $f_{c}=2$.

in firing rate would complicate the interpretation of results as mutual information is known to increase with firing rate [22]). Our calculations using linear response theory demonstrate that the noise spectrum is shaped by the negative ISI correlations without reducing the total noise power in the system. This shaping lowers the noise spectrum at low frequencies, thus enabling better information transfer. Note that our results are different from previous ones on noise shaping [15] as we are considering a nonrenewal process in a single system rather than a network of renewal processes with feedback. The gain $\Delta \mathcal{M}$ in mutual information brought on by ISI correlations was shown to be a nonmonotonous function of signal intensity $\alpha$ and cutoff frequency $f_{c}$. Our results suggest that such a mechanism might be at work in electroreceptive neurons of weakly electric fish. Such mechanisms may exist in other excitable systems.
We thank J.W. Middleton and L. Maler for useful discussions. This research was supported by NSERC and by CIHR.

[1] P. Hänggi, P. Talkner, and M. Borkovec, Rev. Mod. Phys. 62, 251 (1990); L. Gammaitoni, et al., Rev. Mod. Phys. 70, 223 (1998).

[2] T. Heil, I. Fischer, and W. Elsasser, Phys. Rev. A 58, R2672 (1998).

[3] S. B. Lowen and M. C. Teich, Phys. Rev. A 43, 4192 (1991).

[4] F. Rieke et al., Spikes: Exploring the Neural Code (MIT Press, Cambridge, MA, 1996).

[5] A. M. Yacomotti et al., Phys. Rev. Lett. 83, 292 (1999).

[6] D. R. Cox and P. A.W. Lewis, The Statistical Analysis of Series of Events (Methuen, London, 1966).

[7] K. Schäfer et al., Eur. J. Physiol. 429, 378 (1995); J. M. Goldberg and C. Fernández, J. Neurophysiol. 34, 635 (1971); J. M. Goldberg and D. D. Greenwood, J. Neurophysiol. 29, 72 (1966); A. Neiman and D. F. Russell, Phys. Rev. Lett. 86, 3443 (2001); S. Bahar et al., Europhys. Lett. 56, 454 (2001).

[8] R. Ratnam and M. E. Nelson, J. Neurosci. 20, 6672 (2000).

[9] M. J. Chacron et al., Phys. Rev. Lett. 85, 1576 (2000).

[10] M. E. Nelson and M. A. MacIver, J. Exp. Biol. 202, 1195 (1999).

[11] C. D. Geisler and J. M. Goldberg, Biophys. J. 6, 53 (1966); Y. H. Liu and X. J. Wang, J. Comp. Neurosci. 10, 25 (2001).

[12] M. J. Chacron, A. Longtin, and L. Maler, J. Neurosci. 21, 5328 (2001).

[13] R. Azouz and C. M. Gray, J. Neurosci. 19, 2209 (1999).

[14] M. S. Sherwin and A. Zettl, Phys. Rev. B 32, 5536 (1985); K. Wiesenfeld and I. Satija, ibid. 36, 2483 (1987).

[15] D. Maar et al., Proc. Natl. Acad. Sci. U.S.A. 96, 10450 (1999).

[16] G. Gestri, H. A. K. Masterbroek, and W. H. Zaagman, Biol. Cybern. 38, 31 (1980); F. Gabbiani and C. Koch, Neural Comput. 8, 44 (1996); W. Gerstner, Neural Comput. 12, 43 (2000).

[17] A.V. Holden, Models of the Stochastic Activity of Neurones (Springer-Verlag, Berlin, 1976), p. 95.

[18] The power spectrum is given by $S(f)=$ $\left[1+\sum_{i=1}^{\infty}\left(F_{n}(f)+F_{n}(-f)\right)\right] /\langle I\rangle$, where $F_{n}(f)$ is the Fourier transform of the $n$th order ISI density. The $n$th order ISI is given by $t_{n}=\sum_{i=1}^{n}\left(U_{i}+V_{i}\right)$. For model A, we have $t_{n}=n \theta_{0} / \mu+X$ with $X$ having a triangular probability distribution in $[-2 D, 2 D]$. For model $\mathrm{B}, U_{i}$ and $V_{i}$ have uniform probability densities and we have $F_{n}(f)=F_{1}(f)^{n}$.

[19] A. Borst and F. Theunissen, Nat. Neurosci. 2, 947 (1999).

[20] R. Shannon, Bell Syst. Tech. J. 27, 379 (1948); T. Cover and J. Thomas, Elements of Information Theory (Wiley, New York, 1991).

[21] B. Lindner et al. (to be published).

[22] A. Borst and J. Haag, J. Comp. Neurosci. 10, 213 (2001). 